\title{
Breakdown of the quantum Hall effect in periodic and aperiodic antidot arrays
}

\author{
G. Nachtwei, G. Lütjering, D. Weiss, Z. H. Liu, K. von Klitzing, and C. T. Foxon \\ Max-Planck-Institut für Festkörperforschung, Heisenbergstrasse 1, D-70569 Stuttgart, Germany
}

(Received 9 October 1996)

\begin{abstract}
We have studied the breakdown of the quantum Hall effect in $\mathrm{GaAs} / \mathrm{Al}_{x} \mathrm{Ga}_{1-x}$ As heterostructures with antidot arrays as a function of the density and distribution of antidots. For periodic arrays (lithographic antidot diameter $100 \mathrm{~nm}$ ) and periods from 400 to $1000 \mathrm{~nm}$, the breakdown current is systematically reduced with increasing antidot density and determined by the peak value of the local current density. In aperiodic arrays, the breakdown current is markedly lower than in periodic arrays of the same antidot density due to higher local current densities at the same total current. [S0163-1829(97)08811-5]
\end{abstract}

Since 1990, the quantum Hall effect (QHE) (Ref. 1) has been used to realize the unit $\Omega$ of the electric resistance. For high precision measurements with relative uncertainties as low as $10^{-9}$, the sample current should be as high as possible but below the critical current where the nearly nondissipative current flow breaks down. Therefore, a lot of experiments on samples with different material properties ${ }^{2-4}$ and geometries $^{5-7}$ have been performed to understand the physics of the breakdown of the QHE. Since these results are hardly reconcilable within one conclusive model, different mechanisms for the breakdown as intra-Landau-level ${ }^{8}$ and inter-Landau-level transitions, ${ }^{9}$ or a phenomenological description on the basis of electron heating, ${ }^{10,11}$ are still under debate. The breakdown current was found to scale linearly with the sample width for lower electron mobilities. ${ }^{2,3}$ This demonstrates that a nearly homogeneous current distribution exists at currents close to the breakdown in the presence of a high degree of disorder. In contrast, a sublinear increase of the breakdown current with the sample width was found in high-mobility samples, ${ }^{4}$ indicating an inhomogeneous current flow at the breakdown. Additionally, an inhomogeneous current flow leads to a breakdown in distinct local areas of the sample. ${ }^{5-7}$ Thus, the degree of disorder and the homogeneity of the current distribution are important for the breakdown.

In this study, we investigate the influence of periodic and aperiodic antidot arrays on the breakdown of the QHE. The antidots act as artificial repulsive impurities. We show that not only the density, but also the spatial distribution of antidots, is essential for the breakdown. In periodic arrays, the current density is periodically modulated, depending on the antidot spacing and diameter. By investigating the change of the breakdown current with the antidot spacing, we give evidence that the breakdown is determined by the local maximum of current density in the region between adjacent antidots and not by the average of the current density taken over an area including many antidots. In aperiodic arrays, we observe a drastic reduction of the critical currents compared with periodic arrays of the same antidot density. This is due to the inhomogeneous current flow as a result of the irregular spatial variation of the current density. Our results emphasize the importance of local properties on a submicrometer scale for the breakdown of the QHE. However, these results are reconcilable with the recently proposed picture of bootstraptype electron heating over distances of the order of 100 $\mu \mathrm{m},{ }^{12}$ since our measurements of the electron temperature clearly show the relevance of electron heating ${ }^{10-12}$ for the breakdown of the QHE in antidot arrays.

The samples investigated were patterned on a highmobility $\mathrm{GaAs} / \mathrm{Ga}_{x} \mathrm{Al}_{1-x} \mathrm{As}$ wafer with an electron density of $n_{s}=3.04 \times 10^{11} \mathrm{~cm}^{-2}$ and a Hall mobility of $\mu_{H}$ $=1.64 \times 10^{6} \mathrm{~cm}^{2} / \mathrm{Vs}$ corresponding to a mean free path $\ell_{m f p}$ of $15 \mu \mathrm{m}$. The Hall bars (width $w=50 \mu \mathrm{m}$, distance between potential probes $L=50 \mu \mathrm{m}$ ) were defined by photolithography. Two arrays of antidots (periodic with the lattice constant $a$ and aperiodic with the same average antidot spacing $\langle a\rangle$ ) were written on each sample by electron beam lithography and etched by reactive ion etching [see scanning electron microscope (SEM) photograph, inset of Fig. 1]. This arrangement permits an immediate comparison of the QHE breakdown in periodic and aperiodic antidot arrays of the same average antidot density [see inset of Fig. 2(a)]. The lithographic antidot diameter $d_{L}$ is about $100 \mathrm{~nm}$. The distribution function of antidots in the aperiodic arrays is of approximately Gaussian shape with a broadening parameter $\sigma_{a}$ of about $1 / 4\langle a\rangle$ (Fig. 1 for the sample with $\langle a\rangle=600$ $\mathrm{nm})$. Some relevant sample parameters are presented in Table I. The values of the mean free path at zero magnetic field and $T=1.3 \mathrm{~K}$ in the antidot arrays are comparable to the average antidot spacing. Hence, the zero-field resistance is determined by scattering at the antidots.

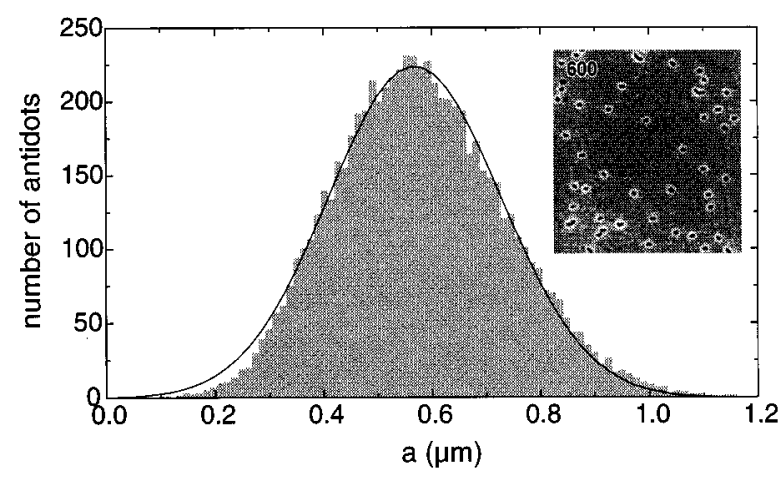

FIG. 1. Distribution function of the antidot number vs nextneighbor spacing $a$ for an aperiodic array with mean spacing $\langle a\rangle=600 \mathrm{~nm}$. Full line: Gaussian distribution of standard deviation $\sigma_{a}=160 \mathrm{~nm}$. Inset: SEM image of the corresponding antidot array. 


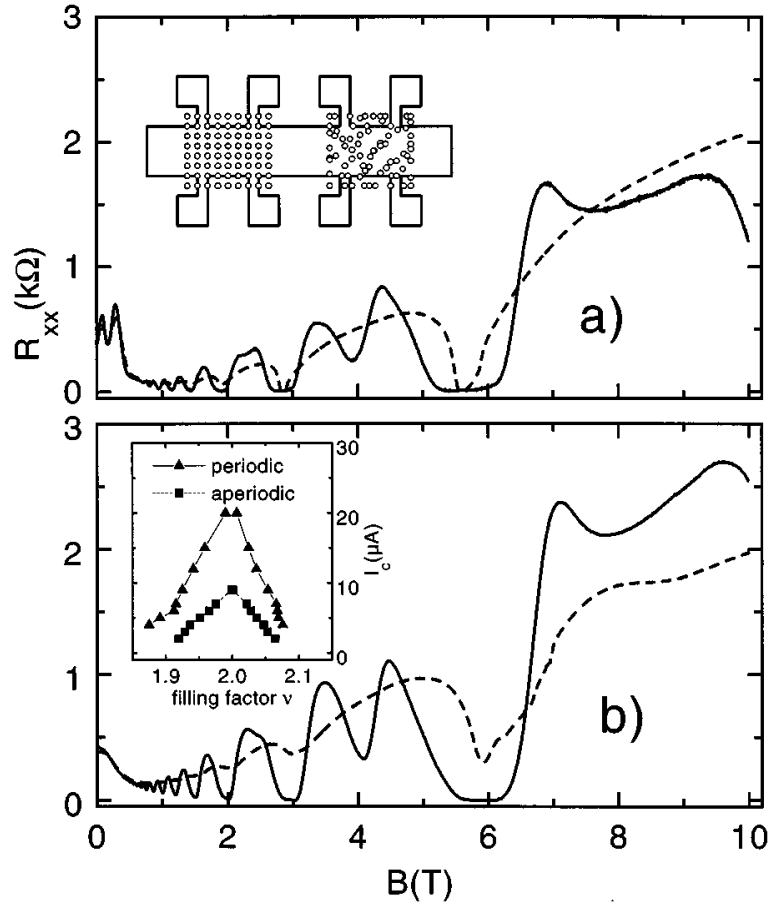

FIG. 2. SdH curves at different sample currents (solid: $1 \mu \mathrm{A}$, dashed: $20 \mu \mathrm{A}$ ) of a periodic array (a) and the corresponding aperiodic array (b) with $a=\langle a\rangle=600 \mathrm{~nm}$ (sample L5a). Inset (a): scheme of the sample geometry (not to scale). Inset (b): filling factor dependence of the critical current $I_{c}$ for the periodic and aperiodic array (sample L5a).

We performed dc measurements $(0.2 \mu \mathrm{A} \leqslant I \leqslant 50 \mu \mathrm{A})$ of the longitudinal resistance $R_{x x}$ and Hall resistance $R_{x y}$ on all samples in magnetic fields $0 \leqslant B \leqslant 10 \mathrm{~T}$ and for temperatures $1.3 \mathrm{~K} \leqslant T \leqslant 24 \mathrm{~K}$. Figure 2 shows a typical set of Shubnikov-de Haas $(\mathrm{SdH})$ curves with the sample current as parameter for sample L5a $(a=\langle a\rangle=600 \mathrm{~nm})$. With increasing current, the plateaus become narrower. For the periodic array, $R_{x x}$ still approaches zero at $B=6 \mathrm{~T}(\mathrm{QH}$ plateau $\nu=2$ ) for $I=20 \mu \mathrm{A}$, in contrast to the aperiodic array (complete breakdown of the QHE). The inset of Fig. 2(b) shows the filling-factor dependence of the critical current $I_{c}$ of the periodic array in comparison with the aperiodic one. For all samples, the critical currents of the periodic arrays are higher

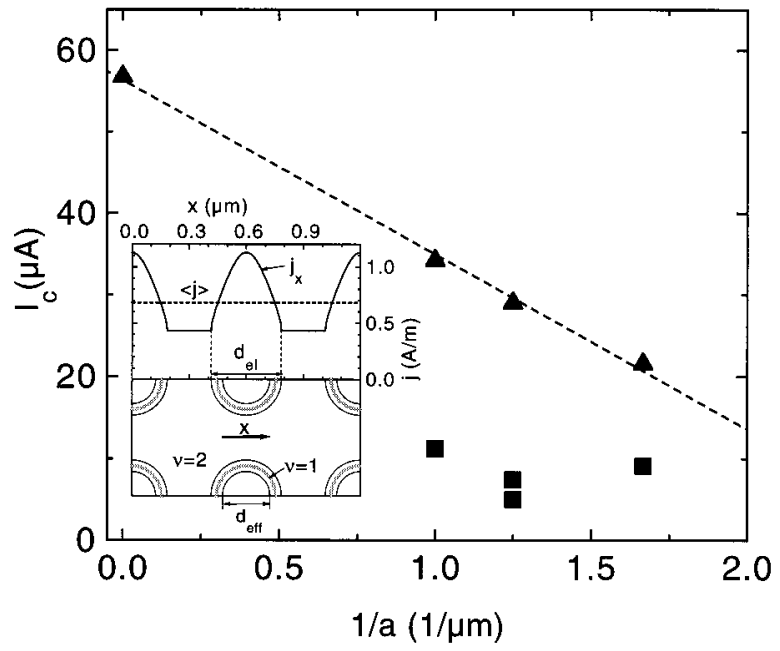

FIG. 3. Critical current $I_{c}(\nu=2)$ vs inverse antidot spacing for periodic $(\boldsymbol{\Delta})$ and aperiodic $(\boldsymbol{\square})$ arrays. The point at $1 / a=0$ refers to the unpatterned sample and fits well into the linear dependence of $I_{c}$ (periodic) on $1 / a$ (dashed line). The inset shows schematically the distribution of the filling factor (lower part) and the current density (upper part) in two adjacent elementary cells of the antidot lattice. The diameters $d_{e f f}$ and $d_{e l}$ include zones of complete depletion and $\nu<2$ (see text), respectively. $j_{x}$ is the current density depending on the position $x$, and $\langle j\rangle$ is the average of $j_{x}$ in current flow direction $x$.

than those of the corresponding aperiodic arrays (see Figs. 2 and 3). For the periodic arrays, the critical current $I_{c}$ decreases linearly with increasing $1 / a$ (Fig. 3). This linear dependence of $I_{c}$ on $1 / a$ can be explained by a simple geometrical argument if the highest local current density $j_{x}^{\max }$ between adjacent antidots reaches an intrinsic critical current density $j_{c}^{0}$ :

$$
j_{c}^{0}=j_{x}^{\max }=\frac{I_{c}(a)}{w-N d_{e l}}
$$

or

$$
I_{c}(a)=I_{c}^{0}\left(1-\frac{d_{e l}}{a}\right)
$$

TABLE I. Sample parameters.

\begin{tabular}{lccccc}
\hline \hline Sample & $\begin{array}{c}\text { antidot } \\
\text { array }\end{array}$ & $\begin{array}{c}a,\langle a\rangle \\
(\mu \mathrm{m})\end{array}$ & $\begin{array}{c}n_{s} \\
\left(10^{15} \mathrm{~m}^{-2}\right)\end{array}$ & $\begin{array}{c}\mu_{H} \\
\left(\mathrm{~m}^{2} / \mathrm{Vs}\right)\end{array}$ & $\begin{array}{c}\ell_{m f p} \\
(\mu \mathrm{m})\end{array}$ \\
\hline L5b & no array & - & 3.04 & 164.0 & 14.9 \\
& aperiodic & 0.8 & 2.94 & 10.7 & 0.96 \\
L4b & periodic & 1.0 & 2.97 & 17.3 & 1.56 \\
& aperiodic & 1.0 & 2.97 & 17.9 & 1.61 \\
L2b & periodic & 0.8 & 2.90 & 11.3 & 1.01 \\
& aperiodic & 0.8 & 2.97 & 9.2 & 0.83 \\
L5a & periodic & 0.6 & 2.70 & 6.1 & 0.53 \\
& aperiodic & 0.6 & 2.85 & 5.1 & 0.43 \\
L4a & periodic & 0.4 & 1.96 & 1.01 & 0.074 \\
& aperiodic & 0.4 & 2.31 & 0.79 & 0.062 \\
\hline \hline
\end{tabular}


where $N=w / a$ is the number of antidots across the sample, $I_{c}^{0}$ is the critical current corresponding to $j_{c}^{0}$ and the sample width $w$, and $d_{e l}$ is the electric antidot diameter. Equation (1) holds for a constant electric Hall field (homogeneous current flow) between the antidot lines.

From the slope of $I_{c}(1 / a)$, a value of $d_{e l}=370 \mathrm{~nm}$ (in the dark) can be estimated. This result is determined by the minimal width $w_{e l}=a-d_{e l}$ of the current path between adjacent antidots. This minimal width occurs in the region between the antidots, where an incompressible strip of constant local density develops under the conditions of the QHE (Ref. 13) (see inset of Fig. 3) that carries the current. The value of $d_{e l}=370 \mathrm{~nm}$ is consistent with the transport data obtained in the sample with $a=\langle a\rangle=400 \mathrm{~nm}$. In the periodic array, the value for $n_{s}$ is markedly lower than the values of both the aperiodic array and the unpatterned sample (see Table I). The QHE is not completely developed in the periodic array with $a=400 \mathrm{~nm}$ due to a reduction of $w_{e l}$ to values close to zero for $a \approx d_{e l}$. The electron densities in Table I indicate an increasing difference of $n_{s}$ of the periodic and aperiodic antidot arrays with decreasing antidot distance. In the periodic arrays, the antidot-potential tails overlap more and more with decreasing lattice constant, leading to a decrease of the local electron density $n_{s}(\mathbf{r})$, especially in the saddle-point region between adjacent antidots. This effect is far less pronounced in aperiodic arrays as the transport current passes through regions of lower local antidot density than in the corresponding periodic arrays.

The assumption, that the breakdown is determined by the highest local current density $j_{x}^{\max }$ between two antidots, yields the same intrinsic critical current density $\left(j_{c}^{0}=1.1\right.$ $\mathrm{A} / \mathrm{m})$ and the same electric antidot diameter $\left(d_{e l}=370 \mathrm{~nm}\right)$ for all samples. This is to be expected, since all samples have identical intrinsic properties (prepared from a homogeneous wafer) and antidot characteristics (identical patterning process). In contrast, the assumption, that the breakdown is determined by a current density $\langle j\rangle$ averaged along the current flow direction, requires a variation of either $d_{e l}$ or $j_{c}^{0}$ by more than $30 \%$ for the different samples.

For the aperiodic arrays, $I_{c}$ shows no clear dependence on $\langle a\rangle$. The lower critical currents observed in aperiodic arrays can be explained by the strong variation of the local antidot density in the aperiodic arrays (Fig. 1), as shown by the following argument: If the current is fixed just slightly below the critical value for the corresponding periodic array $(a=\langle a\rangle)$, the breakdown in the aperiodic array is already complete in all sample regions where $a<\langle a\rangle$ holds. This is due to the local enhancement of the current density between two adjacent antidots according to $j_{\max }=j_{0} a /\left(a-d_{e l}\right)\left(j_{0}\right.$ is the current density outside the antidot array). As a consequence, these regions become dissipative $\left(\sigma_{x x}>0\right)$ and will be avoided by the current flow. ${ }^{7}$ The remaining area available for the current flow will therefore be the total array area reduced by the area of all antidots and the areas in between those antidots where $a<\langle a\rangle$ holds. In contrast, in the periodic array the reduction of area is given by the area of all antidots only. As long as the lattice period $a$ is considerably larger than the antidot diameter $d_{e l}$, the overlap of antidots in the corresponding aperiodic array can be neglected, and the remaining area for the current flow is smaller than in the

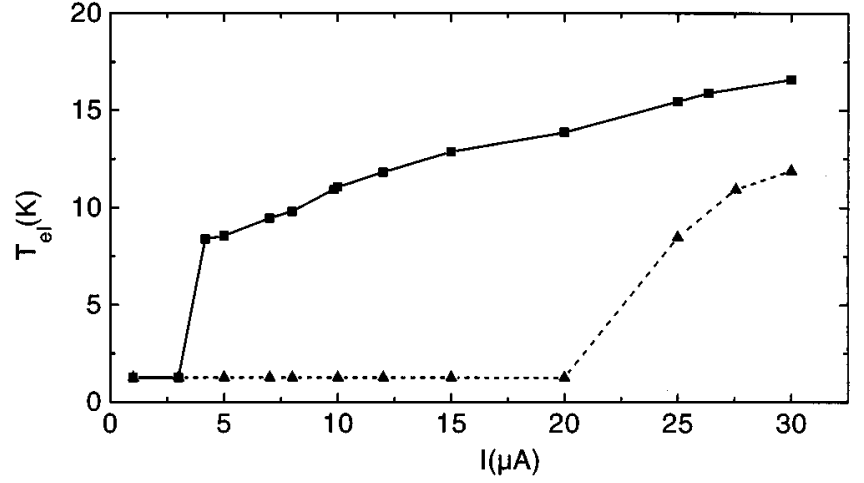

FIG. 4. Temperature $T_{e l}$ of hot electrons vs sample current around the QHE breakdown in a periodic $(\boldsymbol{\Delta})$ and the corresponding aperiodic ( $\boldsymbol{\square})$ array $(a=\langle a\rangle=800 \mathrm{~nm}$, sample L2b).

periodic array. Thus, the breakdown has to be complete in the aperiodic array at current values just below the breakdown in the corresponding periodic array, as observed in the experiments for arrays with $a \geqslant 600 \mathrm{~nm}$. In the aperiodic arrays, the dissipative regions increase with current and form clusters with increasing extension. Simultaneously, the width in between the dissipative clusters decreases and the local current density increases. Hence, the breakdown of the QHE will be triggered in sample regions where the local current density reaches the intrinsic breakdown current density $j_{c}^{0}$.

Due to the percolative nature of the current flow in aperiodic arrays, the local enhancement of the current density which leads to the breakdown can be different from sample to sample, even if $\langle a\rangle$ and $\sigma_{a}$ have the same values. This explains the difference of $I_{c}$ found in two aperiodic arrays of the same value for $\langle a\rangle=800 \mathrm{~nm}$ (samples L5b and L2b). The determination of the QHE breakdown by the highest local current density does not contradict the recently proposed nonlocal electron heating approach, ${ }^{8}$ since the antidot array extends over a length of more than $50 \mu \mathrm{m}$. The electrons can subsequently be heated up while passing the array line by line. The electron heating model explains the QHE breakdown by the balance of energy gain (per unit area and time) due to the current, $\rho_{x x} j^{2}$, and the corresponding loss, due to the relaxation of the heated electrons:

$$
\rho_{x x}\left(T_{e l}\right) j^{2}=\frac{\varepsilon\left(T_{e l}\right)-\varepsilon\left(T_{L}\right)}{\tau_{e}}
$$

where $\varepsilon\left(T_{e l}\right)\left[\varepsilon\left(T_{L}\right)\right]$ is the energy of the electron system (per unit area) at the electron [lattice] temperature $T_{e l}\left[T_{L}\right]$, and $\tau_{e}$ is the energy relaxation time of the heated electrons. Equation (2) takes the temperature dependence of $\rho_{x x}$ into account in terms of the electron temperature only. ${ }^{10,11}$ Using this assumption, we deduced the electron temperatures and their dependence on the current. Figure 4 shows a comparison of the electron temperature in the periodic and the aperiodic arrays of sample L2b $(a=\langle a\rangle=800$ $\mathrm{nm})$. The electron temperatures are determined in the center of the $\mathrm{QH}$ plateau at $\nu=2.0$. Near the breakdown current, the electron temperature rises from the lattice temperature of 1.3 $\mathrm{K}$ to values of about $10 \mathrm{~K}$. In the aperiodic array, the electron 
temperature rises at lower currents than in the periodic array. We attribute this behavior to the higher local current densities in the regions where the current actually flows.

The electron temperatures obtained from transport measurements integrate the sample properties over the area of the array and correspond to the steady state of the hot electron avalanche. ${ }^{12}$ Therefore, the measured electron temperatures may differ considerably from local values on a mesoscopic scale. Further, Eq. (2) is valid only for small differences between electron and lattice temperature, since the electronphonon scattering rate increases with this difference. Hence, the values obtained for the electron temperature can be taken as an estimate only.

To summarize, we have measured the critical currents of the QHE on Hall bars with antidot arrays. In periodic arrays with rather large antidots $\left(d_{L}=100 \mathrm{~nm}, d_{e l}=370 \mathrm{~nm}\right)$, the breakdown is governed by the increase of the maximum local current density between neighboring antidots and not by the globally averaged current density. The critical current is reduced with decreasing spacing between the antidots due to the corresponding increase of the current density between the antidots, which are surrounded by rather large depletion zones. For aperiodic arrays, the breakdown current is strongly reduced in comparison with periodic arrays of the same antidot density, due to the inhomogeneous distribution of the antidots which leads to a strongly inhomogeneous current flow. This is confirmed by measurements of the electron temperature which, in an aperiodic array, rises at lower currents and to higher values than in the corresponding periodic array.

We thank R. R. Gerhardts, B. Farid, and D. Pfannkuche for valuable discussions. Wafers grown at Philips Research Laboratories, Redhill Surrey RH1 5HA, United Kingdom.
${ }^{1}$ K. von Klitzing, G. Dorda, and M. Pepper, Phys. Rev. Lett. 45, 449 (1980).

${ }^{2}$ S. Kawaji et al., Surf. Sci. 305, 161 (1994).

${ }^{3}$ A. Boisen et al., Phys. Rev. B 50, 1957 (1993).

${ }^{4}$ N. Q. Balaban et al., Phys. Rev. Lett. 71, 1443 (1993).

${ }^{5}$ M. E. Cage et al., Phys. Rev. Lett. 51, 1374 (1983).

${ }^{6}$ L. Bliek et al., Semicond. Sci. Technol. 1, 110 (1986).

${ }^{7}$ Ch. Simon et al., Phys. Rev. B 33, 1190 (1986).
${ }^{8}$ P. Středa and K. von Klitzing, J. Phys. C 17, L483 (1984).

${ }^{9}$ L. Eaves and F. W. Sheard, Semicond. Sci. Technol. 1, 346 (1986).

${ }^{10} \mathrm{G}$. Ebert et al., J. Phys. C 16, 5441 (1983).

${ }^{11}$ S. Komiyama et al., Solid State Commun. 54, 479 (1985).

${ }^{12}$ S. Komiyama et al., Phys. Rev. Lett. 77, 558 (1996).

${ }^{13}$ V. G. Burnett, A. L. Efros, and F. G. Pikus, Phys. Rev. B 48, 14365 (1993). 